\title{
Use of Silica-Gold Core Shell Structured Nanoparticles for Targeted Drug Delivery System
}

\author{
Thangaraja Amirthalingam ${ }^{1 *}$, Jegatheesan Kalirajan ${ }^{2}$ and Arunkumar Chockalingam ${ }^{3}$
}

${ }^{1}$ Department of Biotechnology, St Peter's University, Tamilnadu, India

${ }^{2}$ Department of Biotechnology, St Michael Engineering College, Kalayarkovil, India

${ }^{3}$ Department of Chemical Engineering, Annamalai University, Chidambaram, Tamilnadu, India

\begin{abstract}
Drug targeting, using Silica-Gold core carrier particles loaded with antibiotic drugs is an emerging and significant method of disease treatment. Silica core particles were synthesized by Stober's method and it was functionalized with amine groups. The monodispersed Gold particles were prepared by conventional citrate reduction method. Both functionalized Silica and monodispersed Gold nanoparticles were combined and Silica-Gold core nanoshells was prepared in the range of $60-110 \mathrm{~nm}$. The morphology, elemental composition of the core nanoshell was characterized by UV-Visible spectroscopy, FT-IR spectroscopy, Scanning Electron Microscope (SEM), Energy Diffusive Spectroscopy (EDX) and X-ray Diffraction analysis. The antibiotic Gentamycin was loaded onto these Silica-Gold core nanoshell carriers and the drug release profile was observed.
\end{abstract}

Keywords: Drug loading; Gentamycin; Silica-Gold core nanoshell; Citrate reduction method

\section{Introduction}

During the last decade, owing to the emergence of a new generation of high technology materials, the number of research groups involved in nanomaterials has increased exponentially [1]. Core-shell particles are very important because of their applications in areas such as catalysis, cancer diagnosis and sensors [12]. Nanoshells are obtained by removing the cores of these particles or by over coating the inert core with a thin coat [2]. Core shell nanoparticles consist of concentric particles, in which particles of one material are coated with a thin layer of another material using specialized procedures [3]. The existing drug delivery systems can be categorized into two groups, namely the polymer- and lipid-based systems, in which the drugs were incorporated into or attached to the polymer or lipid [4].

In the recent past there has been considerable interest in nanosystems such as Gold nanoparticles, carbon nanotubes, etc. for drug delivery applications. To date, Gold nanoshells offer the added features of high biocompatibility and bioconjugation to antibodies via long-standing protocols adapted from Gold colloid bioconjugate chemistry, their near-infrared optical properties make them an ideal, if not unique, vehicle for a whole blood immunoassay [5]. Nanoshells made of oxides such as Silica and Titania find application in the field of drug delivery. The outer surface of these shells can be used for attaching antibodies so that the Silica shell-antibody complex can be used for targeted drug delivery in biological systems [6].

Silica-Gold core nanoshell in drug delivery system may offer plenty of advantages over conventional dosage forms, which improves efficacy, reduced toxicity, enhanced biodistribution and reduces patient's compliance. The particles have the ability to conjugate the molecules without affecting the core and also can be used to encapsulate the drugs [7]. The present study Silica-Gold core nanoshells were synthesized and characterized for use of targeted drug delivery.

\section{Materials and Methods}

All reagents (expect water) were purchased from the Himedia and used without modification. Deionized water was purified by milli-Q water system (Millipore Corporation) and filtered through various filter to remove any impurities.

Silica and Gold nanoparticles were separately synthesized by Sol-gel method and Silica particles were coated with respective shell materials $(\mathrm{Au})$ in multistep process.

Silica nanoparticles were synthesized by well known Stober's method [8] Different size of monodispersed Silica nanoparticles of various sizes was synthesized by changing the concentration of ethanol and ammonium hydroxide. The Silica nanospheres were synthesized by hydrolysis of $1.2 \mathrm{ml}$ of tetraethoxysilane in a $10 \mathrm{ml}$ of ethanol, 3 $\mathrm{ml}$ of water and $0.75 \mathrm{ml}$ of ammonium hydroxide and solutions was stirred at room temperature for 3 hours. After completion of reaction, the Silica nanoparticles were collected by ultrafiltration. Since Gold had very little affinity towards Silica, so silane coupling agent was used to functionalize the Silica nanoparticles. The Silica nanoparticles subsequently functionalized by particles dissolved in ethanol followed by the addition of $0.5 \mathrm{ml}$ of 3 -aminopropyltriethoxysilane (APS). The mixture was vigorously stirred at $60^{\circ} \mathrm{C}$ for $15 \mathrm{hrs}$ to promote covalent binding of APS moieties onto the Silica particles [9]. The amine functionalized Silica particles were centrifuged on R-24 refrigerated centrifuge (REMI) at $2000 \mathrm{rpm}$ for $1 \mathrm{hr}$. The particles were thrice redispersed in $100 \mathrm{ml}$ of ethanol to remove unattached APS and other contaminants.

The Gold nanoparticles were synthesized through the common techniques of citrate reduction, which has been described in detail. The preparation of Gold nanoparticles by dissolve tetrachloroaurate

*Corresponding author: Thangaraja Amirthalingam, Department of Biotechnology, St Peter's University, Avadi, Chennai, Tamilnadu, India, Tel: +91-9965256568; E-mail: athangaraja@gmail.com

Received August 29, 2011; Accepted November 14, 2011; Published November 16, 2011

Citation: Amirthalingam T, Kalirajan J, Chockalingam A (2011) Use of SilicaGold Core Shell Structured Nanoparticles for Targeted Drug Delivery System. J Nanomedic Nanotechnol 2:119. doi:10.4172/2157-7439.1000119

Copyright: (C) 2011 Amirthalingam T, et al. This is an open-access article distributed under the terms of the Creative Commons Attribution License, which permits unrestricted use, distribution, and reproduction in any medium, provided the original author and source are credited. 
$(180 \mathrm{mg})$ and trisodium citrate $(600 \mathrm{mg})$ in water $(590 \mathrm{ml})$, then the mixture was kept in water bath and the temperature maintained at $80^{\circ} \mathrm{C}$ for 30 minutes. After that Gold colloid solution was cooled to room temperature and store at $5^{\circ} \mathrm{C}[10]$.

The synthesis of Silica-Gold core nanoshell by attachment of clusters to amine-functionalized Silica particles to the Gold nanoparticles by Osterloh method [11]. $10 \mathrm{ml}$ of the $\mathrm{SiO}_{2}$.APS particles in ethanol were taken and washed with $20 \mathrm{ml}$ of water, then after redispersed in 20 $\mathrm{ml}$ of water and $\mathrm{pH}$ maintained at 5.0 . To that $1.0 \mathrm{ml}$ of Silica-APS solution was added into $20 \mathrm{ml}$ of Gold-citrate solution by drop wise, with stirring. After 5 minutes, the solution was centrifuged at $3000 \mathrm{rpm}$ and the deep red solid was collected, then it first washed with $20 \mathrm{ml}$ of water and then washed with $20 \mathrm{ml}$ of methanol.

\section{Characterization methods}

Different types of nanoparticles were characterized through plasmon spectrum analysis (UV-Visible Spectroscopy), Scanning Electron Microscopy (SEM) analysis, FT-IR (Fourier Transform Infra Red) spectrum studies and EDX (Energy Diffusive X-ray analysis.

UV-Visible spectra were obtained using Hitachi UV-Visible spectrometer over the wavelength range of $200-800 \mathrm{~nm}$. This is used to find out the plasmon peak to detect the presence of specific molecule in the sample.

The FT-IR spectrum was recorded on Perkin Elmer FTIR 1650 spectrophotometer at ambient temperature using $\mathrm{KBr}$ pellet method. The pellet was scanned at 16 scans at wave number range of 400 $4000 \mathrm{~cm}^{-1}$

For SEM studies dried Silica nanoparticles, Gold nanoparticles as well as Silica-Gold core nanoshell particles were coated with gold to improve the electrical conductivity. The samples were examined at a magnification of 20000 to $150000 \mathrm{X}$ to find out the shape and size of the particles.

Elemental analysis of Silica-Gold core nanoshell was done by Energy Diffusive X-ray (EDX) analysis for the presence of core material in the sample. The strong signal from the sample is observed and value is noted.

The crystallographic study was performed on the synthesized Silica-Gold core nanoshell by X-ray diffractometer. The sizes of the nanoparticles were calculated using Bragg's law.

\section{Drug loading and releasing pattern}

Loading of drug was carried out by the addition of $4 \mathrm{ml}$ of Gentamycin to $20 \mathrm{ml}$ of nanoshell and the mixture was stirred for $24 \mathrm{hrs}$ [12]. At fixed time intervals, the nanoshells were separated from the liquid by ultrafilteration and optical density of residual Gentamycin in the supernatant was measured at $285 \mathrm{~nm}$ by UV-Visible spectrophotometer. Drug loading was determined as the difference between the initial Gentamycin concentration and the concentration of Gentamycin in the supernatant.

Drug loaded nanoshell carriers were then separated through ultrafilteration and air dried. Drug release profile was obtained by dissolving the dried drug loaded nanoshells in 5ml PBS buffer at $37^{\circ} \mathrm{C}$. Concentration of Gentamycin released in the aqueous solution was observed at fixed time interval at $285 \mathrm{~nm}$ by UV - Visible spectrophotometer.

\section{Results}

Core shell type Silica-Gold nanoparticles clusters form by reaction of 3-aminopropyltriethoxysilane (APS) - modified Silica spheres with a solution of citrate-coated Gold nanoparticles at pH 5.0. The Silica-Gold nano coreshells were isolated by ultrafiltration and stored as dispersed in methanol.

The characterization of all type of nanoparticles was studied by UV-Visible spectroscopy. The absorbance spectrum of both Stobers Silica nanoparticles absorbed at $210 \mathrm{~nm}$ and functionalized Silica nanoparticles revealed the presence of peak at $220 \mathrm{~nm}$, a specific peak for Si-O-Si bond and it confirms the presence of Silica nanoparticles. The optical absorption of pure Gold nanoparticles solutions was found at $525 \mathrm{~nm}$ as shown in Figure 1. The UV-Visible spectra of SilicaGold core nanoshell revealed a peak at $510 \mathrm{~nm}$ because it shifts the wavelength slightly from red to blue due to binding of Gold with Silica and form the coreshell.

FTIR is used to identify types of chemical bonds (functional groups) between the atoms or molecules. The wavelength of light absorbed is characteristic of the chemical bond present in the chemicals. By interpreting the infrared absorption spectrum; the chemical bonds in a molecule can be determined. FTIR spectra of pure compounds are generally so unique that they are like a molecular fingerprint.

FTIR results of the present study in Figure 2 shows the spectrum of Silica, Functionalized Silica; Gold and Silica-Gold core nanoshell particles. Silica and Silica-Gold core nanoshell particles showed a peak at $1193.85 \mathrm{~cm}^{-1}$ which can be assigned to $\mathrm{Si--O}--\mathrm{Si}$ bond and the peak at $945.05 \mathrm{~cm}^{-1}$ can be attributed to $\mathrm{Si}--\mathrm{OH}$ bond while for functionalized silica particles peak at $3269.12 \mathrm{~cm}^{-1}$ can be assigned to $\mathrm{NH}$ bond. In the spectrum of the functionalized silica particles coated with gold the intensity of $\mathrm{Si--O}--\mathrm{Si}$ and $\mathrm{Si--OH}$ peaks has been reduced significantly. This indicates the presence of gold shell in silica particles.

Figure 3 represents a Scanning electron micrograph (SEM) of Silica nanoparticles, Gold nanoparticles and Silica-Gold nanoshells. It can be seen that Silica particles have well defined shapes. The sizes of the Silica particles have a diameter of $30-70 \mathrm{~nm}$ in Figure 3a. The sizes of the Gold nanoparticles were in the range of $10-40 \mathrm{~nm}$ in Figure $3 \mathrm{~b}$ and Silica-Gold nanoshell sizes in the range of $60-110 \mathrm{~nm}$ in Figure 3c. Figure 3d shows the Energy Diffusive X-ray (EDX) spectrum recorded in the particle mode from one of the densely populated nanoshell.

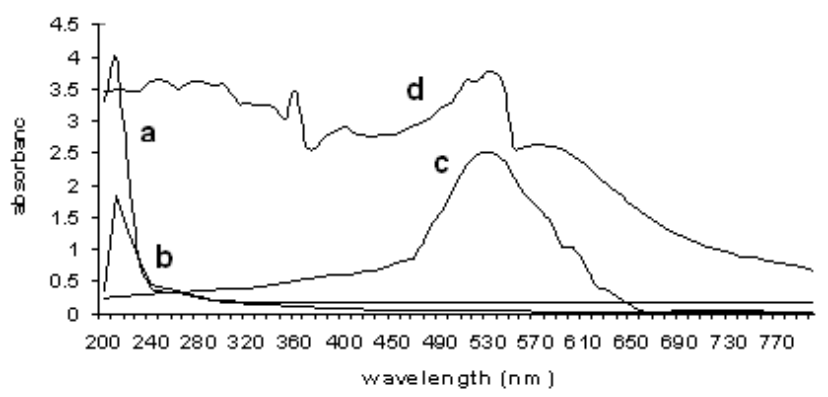

Figure 1: Comparative analysis of UV-Visible spectrum of nanoparticles (a) Absorption spectrum of Silica nanoparticles (b) Absorption spectrum of Functionalized silica nanoparticles (c) Absorption spectrum of Gold nanoparticles (d) Absorption spectrum of Silica-Gold core nanoshell. 


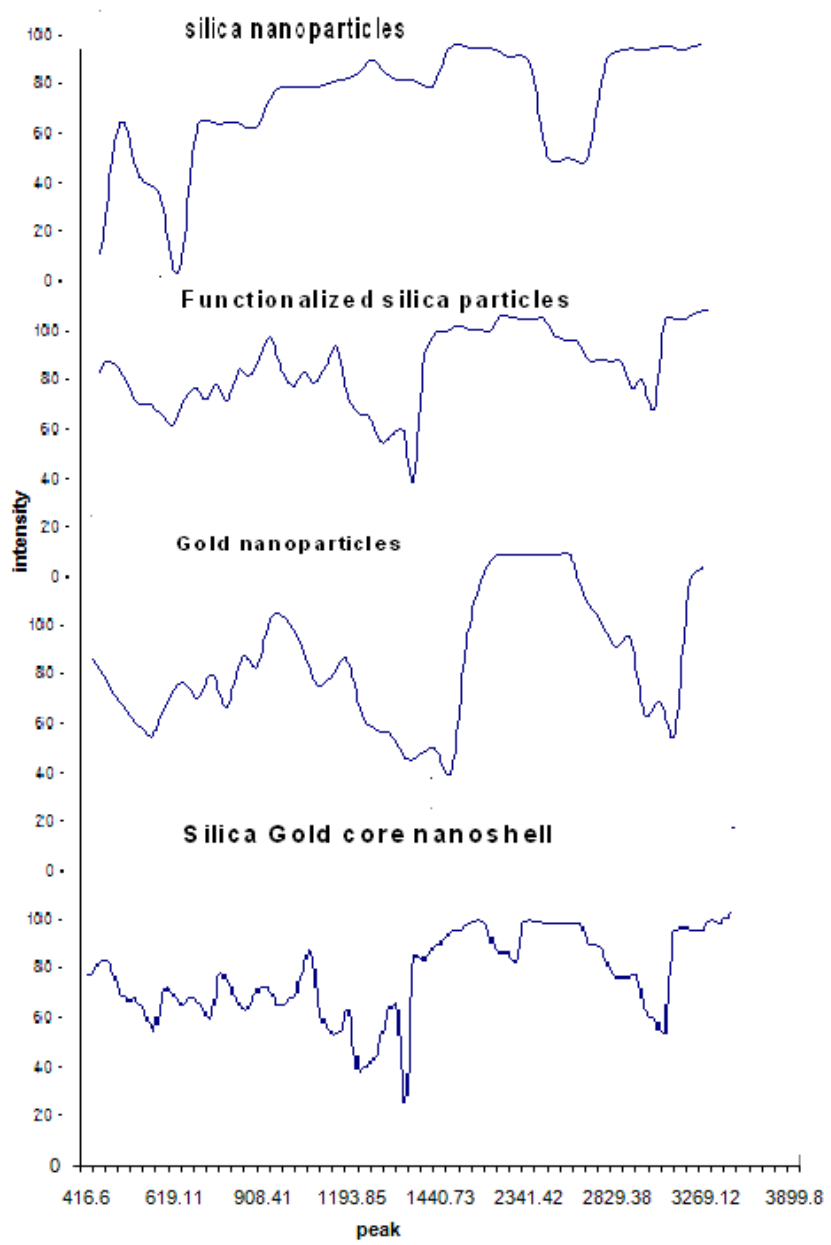

Figure 2: FT-IR spectra of Silica, Gold and Silica-Gold core nanoshell particles.

Strong signals from the Silica and Gold atoms in the nanoparticles were observed.

The elemental analysis measurements show that the Silica-Gold core nanoshell composed of metallic $\mathrm{Au}$ and $\mathrm{Si}$ and some other contaminants also present.

The X-ray diffraction pattern taken from Silica-Gold core nanoshell is shown in Figure 4. All the peaks were corresponding to the SilicaGold diffraction. The mean values for Silica-Gold core nanoshell was calculated as $32 \mathrm{~nm}$ based on Bragg's law. The X-ray Diffraction pattern of Silica is hidden under the pattern of Gold due to overlapping of their diffraction peaks at $2 \Theta=56.66 ; 64.82 ; 75 ; 77.8517$. The numbers of diffraction patterns of $\mathrm{Si}--\mathrm{OH}$ were observed in the $\mathrm{X}$ - ray Diffraction spectrum indicating that the Silica nanoparticles were well protected by the Gold shell. The diffraction studies confirm a high degree of crystallinity and uniformity in the particles.

The drug loaded Silica-Gold core nanoshell has potential application in drug carrier and delivery system. The Silica--Gold core nanoshell mixed with Gentamycin solution and the amount of Gentamycin absorbed by core shell was calculated. The drug Gentamycin loading and releasing pattern to Silica--Gold core nanoshell as shown in Figure 5. Initially, the adsorption of Gentamycin was rapid by nanocarriers, the adsorption rate slow down and reached saturation level. In $30 \mathrm{~h}$ of loading $87 \mu \mathrm{g}$ are loaded per mg of Silica - Gold core nanoshell.

The drug release pattern of nano carriers was observed in Figure 6. It is apparent from Figure 6 there is a continuous release of drug up to $30 \mathrm{~h}$ beyond which slows down. A maximum of $25 \%$ of adsorbed drug was released in $90 \mathrm{~h}$ from nano carriers.

\section{Discussion}

For pure Gold nanoparticles, Plasmon shift of pure Gold nanoparticles has been observed at $525 \mathrm{~nm}$ and absorption spectrum of MTMOS sol-gel: Similar shift of Plasmon band for Gold nanoparticles solution has been observed at $524 \mathrm{~nm}$ [12]. The results of the present study were also referred to Pd-coated Silica nanoparticles, the plasmon peak observed at $520 \mathrm{~nm}$ and THPC Gold nanoparticles have plasmon absorption peak at $520 \mathrm{~nm}$ [13].
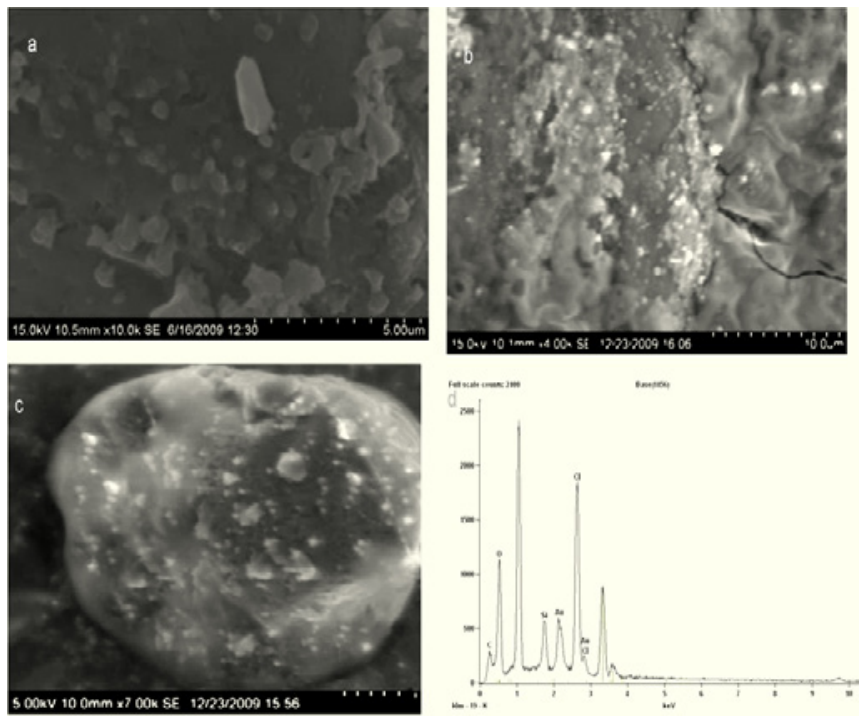

Figure 3: SEM micrograph of different nanoparticles (a) Silica nanoparticles (b) Gold nanoparticles (C) Silica-Gold core nanoshell (d) EDS spectrum SilicaGold core nanoshell.

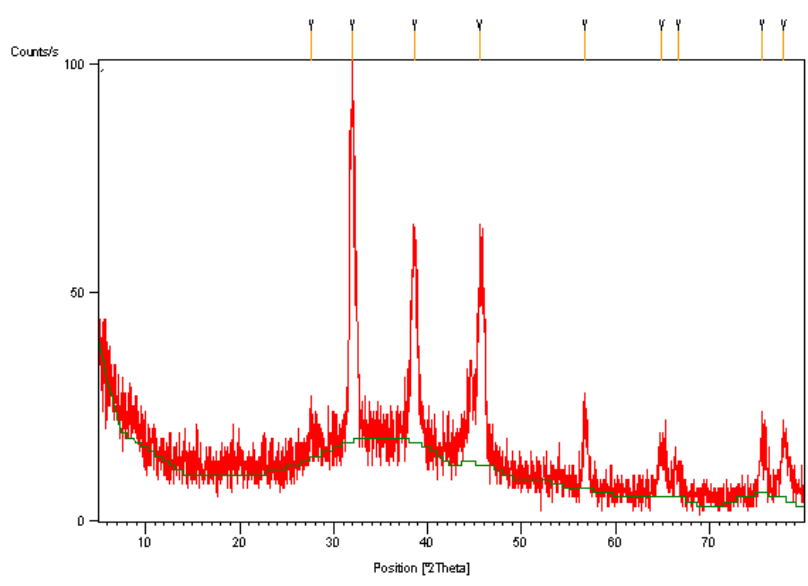

Figure 4: X-ray diffraction spectrum of Silica-Gold core nanoshell. 


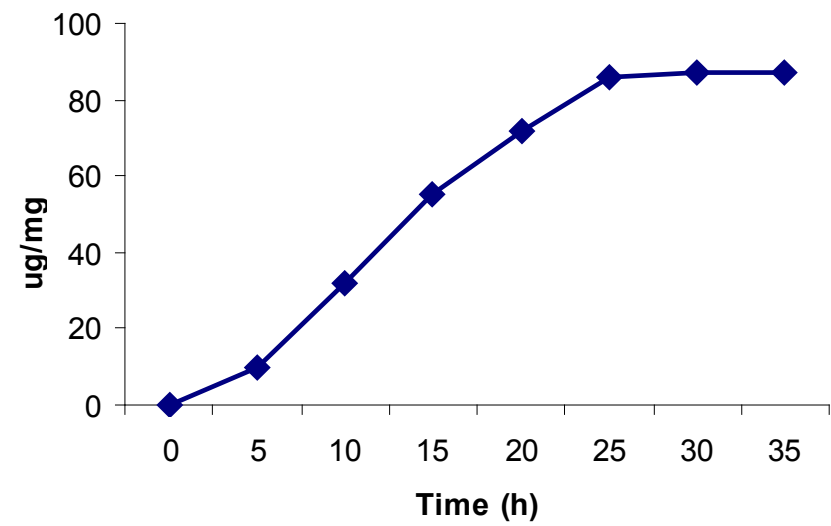

Figure 5: Drug Gentamycin loading on to Silica-Gold core nanoshell.

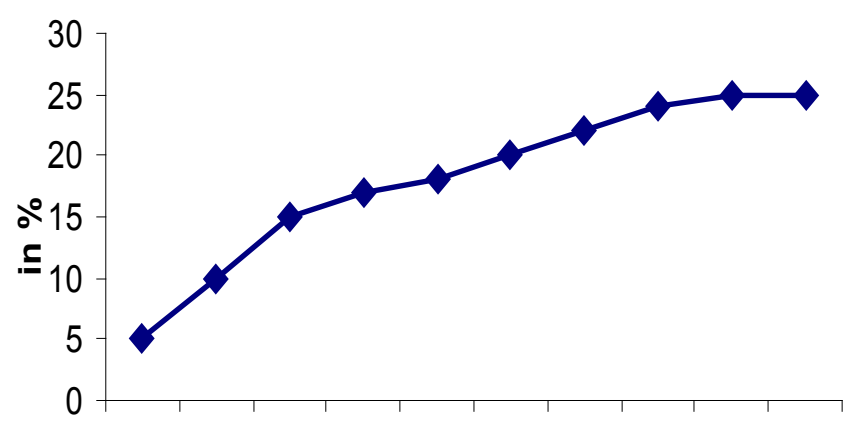

$\begin{array}{llllllllll}10 & 20 & 30 & 40 & 50 & 60 & 70 & 80 & 90 & 100\end{array}$ Time (h)

Figure 6: Drug Gentamycin releasing pattern from Silica-Gold core nanoshell.

\begin{tabular}{|l|l|l|}
\hline Pos. $\left[{ }^{\circ} \mathbf{2 T h}.\right]$ & FWHM $^{\circ}{ }^{2}$ Th. & d-spacing $[\AA]$ \\
\hline 27.5748 & 0.3149 & 3.23488 \\
\hline 31.9434 & 0.2362 & 2.80175 \\
\hline 38.5700 & 0.7085 & 2.33428 \\
\hline 45.5367 & 0.3936 & 1.99205 \\
\hline 56.6618 & 0.4723 & 1.62452 \\
\hline 64.8822 & 0.7872 & 1.43716 \\
\hline 66.5903 & 0.6298 & 1.40438 \\
\hline 75.5772 & 0.7872 & 1.25816 \\
\hline 77.8517 & 0.7680 & 1.22598 \\
\hline
\end{tabular}

Table 1: Data for X-ray diffraction spectrum of Silica-Gold core nanoshell.

When an aqueous solution of Gold nanoparticles was mixed with an ethanolic solution of APS - Functionalized Silica nanoparticles, the Gold nanoparticles become immobilized on the surfaces of the Silica nanoparticles; the coverage of Gold nanoparticles is 30\%, consistent with the reported coverage of charged nanoparticles on planar amine functionalized surfaces [13]. The attachment was sufficiently strong, so that the Gold nanoparticles remain attached to the Silica nanoparticles.

The antimicrobial drugs loaded nano core shell advantage to act as vehicle for drug delivery, because it was biocompatible and protective surfaces. Also the Gold coated Silica has more hydrophobicity, which helps for the penetration of drugs into bacterial cells [14].

The pore size and environment can be tailored to selectively store different molecules of interest [15], while the size and scope of the particles can be turned to maximized cellular uptake. Unlike polymerbased nanoparticles, these robust inorganic materials tolerated many organic solvents [16]. Silica based particles have been successfully used as drug delivery vectors [17], gene transfect ion agents [18] and carriers of molecules [19].

This report demonstrates the synthesis of Silica-Gold core nanoshell on the surface of Silica nanoparticles cores. The method described herein enables the synthesis of core-shell particles having dimensions substantially greater than $60-100 \mathrm{~nm}$. Monodispersed particles (Silica and Gold) with various core sizes $30-200 \mathrm{~nm}$ were obtained by this reduction method. All the analyses collectively confirm the formation of Silica-Gold core nanoshell in sizes ranging from $10-100 \mathrm{~nm}$. The results of drug loading and releasing show that a significant amount of Gentamycin could be loaded onto the Silica-Gold core nanoshell. The amount of Gentamycin loaded in Silica-Gold core nanoshell is observed as $87 \mu \mathrm{g} / \mathrm{mg}$. The Gentamycin attached to the Silica-Gold core nanoshell through amine group of Gentamycin with the Gold particles. $\mathrm{NH}_{2}$ group of doxorubicin drug was involved in binding of drug on to the gold nanoparticles surface [20]. NH group involved to finding 5-Flurouracil on to Gold nanoparticles surface area [21]. The drug release can be explained by the covalent conjugation model postulated by Ringsdorf [22], where break of Au-Gentamycin coordinate linkers results in the release of drug from core shell. In conclusion, a Silicashell based drug delivery system can be achieved by incorporating Gentamycin in Silica-Gold core nanoshell. Future studies aiming to find out the antibacterial activity of Gentamycin drug loaded SilicaGold core nanoshell.

\section{Acknowledgement}

The Authors thank Tamilnadu State Council for Science and Technology (TNSCST), Chennai for financial support for this research project.

\section{References}

1. Deepika K, Suchita K, Kulkarni SK (2007) Synthesis and characterization of Silica- Gold core shell (SiO@Au) nanoparticles. J Physics 69: 277-283

2. Saran $P$, John DB, Son-Jong H, Alexander K (2005) Investigation of the CoreShell Interface in Gold@Silica Nanoparticles: A Silica imprinting approach. Langmuir 21: 12348-12356

3. Jie Lu, Monty L, Sean S, Tian X, Michael K, et al. (2007) Mesoporous Silica Nanoparticles for Cancer Therapy: Energy-Dependent Cellular Uptake and Delivery of Paclitaxel to Cancer cells. Nanobiotechnology 3: 89-95

4. Nagarajan S, Yong Z (2008) Use of Core/Shell Structured Nanoparticles for Biomedical Applications. Biomedical Engg 1: 34-42

5. Hirsch LR, Jackson JB, Lee A, Halas NJ, West JL (2003) A Whole Blood Immunoassay Using Gold Nanoshells. Anal Chem 75: 2377-2381

6. Rosemary MJ, Ian ML, Pradeep T (2006). Investigation of the Antibacteria properties of Ciprofloxacin@SiO . Langmuir 22: 10125-10129

7. Zhou W, Peng GT, Lei S, Daniela C, Minghui Y, et al. (2005) Drug-Loaded, magnetic, hollow silica nanocomposites for Nanomedicine. Nanomedicine 1 : 233-237

8. Stöber W, Fink A, Bohn E (1968) Controlled growth of mono-disperse silica spheres in the micron size range. J Colloid Interface Sci 26: 62-69

9. Kim JH, Chung HW, Lee TR (2006) Preparation and Characterization of Palladium Shell with Gold and Silica Cores. Chem Mater 18: 4115-4120

10. Kimling J, Maier M, Okenve B, Kotaidis V, Ballot H, et al (2006) Turkevitch method for Gold nanoparticles synthesis revisited. J Phys Chem 110: 15700 15707

11. Osterloh F, Hiramatsu H, Rhiannon P, Ting G (2004) Alkanethiol- Induced Structural Rearrangements in Silica-Gold Core shell type Nanoparticles Clusters: An opportunity for Chemical sensor Engineering. Langmuir 20: 55535558 
Citation: Amirthalingam T, Kalirajan J, Chockalingam A (2011) Use of Silica-Gold Core Shell Structured Nanoparticles for Targeted Drug Delivery System. J Nanomedic Nanotechnol 2:119. doi:10.4172/2157-7439.1000119

Page 5 of 5

12. Kefalides PT (1998) New methods for drug delivery. Annals Internal Medicine 128: $1053-1055$

13. Westcott SL, Oldenburg SJ, Lee TR, Halas JH (1998) Formation and Adsorption of clusters of gold nanoparticles onto Functionalized Silica nanoparticles surfaces. Langmuir 14: 5396-5401

14. Darryl NW, Sheryl HE, Tracey RPH (2006) Evaluation of the microbial growth response to inorganic nanoparticles. J Nanobiotechnology 4: 1-8

15. Munoz B, Ramila A, Diaz I, Perez-Pariente J, Vallet-Regi M (2003) MCM-41 Organic modification as drug delivery rate regulator. Chem Mater 15: 500-503

16. Stein A, Melde BJ, Schroden RC (2000) Hybrid Inorganic-Organic Mesoporous Silicates-Nanoscopic Reactors Coming of Age. Adv Mater 12: 1403

17. Arruebo M, Fernandez PR, Ricardo IM, Jesus S (2007) Magnetic nanoparticles for drug delivery. Nanotoday. 2: 22-32
18. Radu DR, Lai CY, Jeftinija K, Rowe EW, Jeftinija S, et al. (2004) A polyamidoamine dendrimet- capped mesoporous Silica nanospheres based gene transfection reagent. J Am Chem Soc 126: 13216-13217

19. Lai CY, Jeftinija DM, Jeftinija K, Xu S, Jeftinija S, et al. (2003) A Mesoporous silica nanospheres-based carrier system with chemically removable CdS nanoparticle caps for stimuli-responsive controlled release of neurotransmitters and drug molecules. J Am Chem Soc 125: 4451-4459

20. Abdiche YN, Myszka DG (2004) Probing the mechanism of drug/lipid membrane interactions using Biacore. Anal Biochem 328: 233-243

21. Huang X, El-Sayed IH, Qian W, El-Sayed MA (2006) Cancer cell imaging and photothermal therapy in the near-infrared region by using Gold nanorods. J Am Chem Soc 128: 2115-2120

22. Ringsdorf H, Haussling L, Schmitt F-J, Knoll W (1991) Biotin-functionalized self-monolayers on gold: surface plasmon optical studies of specific recognition reactions. Langmuir 7: 1837. 